\title{
FLUTUAÇÃO POPULACIONAL DE PRAGAS E SEUS INIMIGOS NATURAIS EM SOJA NO PROJETO RIO FORMOSO - FORMOSO DO ARAGUAIA - TO, BRASIL.
}

\author{
Julcemar DIDONET ${ }^{1}$, Daniel de B. FRAGOSO², Joenes M. PELUZIO', Gil R. \\ dos SANTOS'.
}

RESUMO - Neste estudo, desenvolvido na entressafra do arroz (Julho-Outubro) de 1995, no Projeto Rio Formoso, municipio de Formoso do Araguaia -TO, com o objetivo de verificar o comportamento da população das pragas e seus inimigos naturais em soja, foram realizados levantamentos semanais com o método do pano de batida, em três cultivares de soja de ciclo médio: EMGOPA 308, DOKO RC e EMBRAPA 31 (BR 81). Entre os percevejos fitófagos somente foi constatada a ocorrência de Piezodorus guildinii, com picos populacionais no período de enchimento de grãos, porém não atingindo o nivel de controle. As lagartas desfolhadoras encontradas foram Anticarsia gemmatalis, Hedylepta indicata e Chrysodeixis includens, em ordem decrescente de abundância. Dos coleópteros desfolhadores, a espécie encontrada em maior abundância foi Cerotoma sp. com picos populacionais próximos ao periodo reprodutivo, diferindo significativamente entre as cultivares. Dentre os inimigos naturais, foi verificada a ocorrência, em maior abundância de Cycloneda sanguinea (Coleoptera, Coccinellidae), Geocoris sp. (Heteroptera, Lygaeidae) e Lebia sp. (Coleoptera, Carabidae).

Palavras-chave: Dinâmica Populacional, Insetos-praga, Inimigos Naturais.

Population Dinamic of Soybeans Pests and Their Natural Enemies in Rio Formoso Project - Formoso do Araguaia - To, Brazil.

ABSTRACT - This experiment was carried out at the Experimental Station of the Universidade do Tocantins in Formoso do Araguaia-TO, to study the population dinamics of soybean pests and their natural enemies. The weekly surveys were made using the plant-shaking method, in three cultivars of the medium cycle, during the period from July to October 1995, installed in a randomized block design, with four blocks. The majority of species collected ocurred in low numbers, and from the stink bug complex, only ocurred the species Piezodorus guildinii, (Heteroptera, Pentatomidae), with population peaks in the stage of grain filling. The defoliator catterpilars were found Anticarsia gemmatalis (Lepidoptera, Noctuidae), Hedylepta indicata (Lepidoptera, Pyralidae) and Chrysodeixis includens (Lepidoptera, Noctuidae), in decrease order of the abundance. Cerotoma sp., was the most abundant defoliator beetle found in the extension of the culture development cycle, with populations peaks closely the reprodutive stage. Among the natural enemies collected, Cicloneda sanguinea (Coleoptera, Coccinellidae); Geocoris sp. (Heteroptera, Lygaeidae) and Lebia sp. (Coleoptera, Carabidae) were the most abundants.

Key-Words: Population Dinamic, Soybeans Pests, Natural enemies

\section{INTRODUÇÃO}

No complexo ecossistema formado pela cultura da soja, diversas espécies de insetos são encontradas, porém poucas são consideradas efetivamente como pragas-chave. Dentre estas, Piezodorus guildinii Westwood, 1837, Euschistus heros Fabricius, 1798, Nezara viridula Linnaeus, 1758 (Heteroptera: Pentatomidae) e Anticarsia gemmatalis Hübner, 1818

Fundação Universidade do Tocantins - UNITINS, Campus Universitário de Gurupi, Al. Madrid, Qd 6, Lt 8/9, CEP 77410-470, Gurupi-TO.

2 Acadêmico de Agronomia, Fundação Universidade do Tocantins, Gurupi-TO. 
(Lepidoptera: Noctuidae) são referidas como pragas principais e de maior importância econômica para a cultura, com seus danos refletindo direta ou indiretamente na produção e/ou na qualidade dos grãos e sementes (Gazzoni et al., 1988). Outras espécies são consideradas como pragas secundárias e de menor importância econômica, pois ocorrem em menor abundância e somente causam danos significativos para a cultura em condições especiais. Este complexo ainda possui grupos de insetos benéficos, que atuam como agentes de controle biológico. Alguns desses agentes são tão eficazes que, sob determinadas condições, mantém as populações de insetos-praga abaixo do nível de dano econômico, dispensando, assim, a necessidade do controle químico (Borkert et al., 1994).

Para o controle racional de insetos-praga necessita-se de métodos mais eficientes em substituição aos métodos tradicionais de controle, e neste contexto o conhecimento das espécies que são consideradas pragaschave e seus inimigos naturais, bem como as flutuações populacionais são parâmetros de grande importância na adoção do manejo integrado de pragas (Gazzoni et al., 1988). Na cultura da soja vários estudos têm sido feitos com esta finalidade, nos vários Estados do Brasil (Corrêa et al., 1977; Lorenzato et al., 1979; Prado et al., 1982; Salvadori \& Gomez, 1982; Leite \& Lara, 1985).

Na região produtora do Projeto Rio Formoso há necessidade de informações referentes as principais pragas e inimigos naturais, bem como a flutuação populacional correlacionada com o estádio de desenvolvimento da cultura. Tais informações são de grande importância na aplicação de táticas e estratégias de controle, sendo, portanto, de grande contribuição para o setor agrícola, além de que o Estado do Tocantins apresenta grande potencial agrícola e perspectiva de expansão da cultura da soja. O presente trabalho foi realizado com o objetivo de estudar a dinâmica populacional das pragas e seus inimigos naturais em soja, visando a obtenção de dados para auxiliar o manejo integrado de pragas nesta cultura.

\section{MATERIAL E MÉTODO}

$\mathrm{O}$ experimento foi conduzido na Estação Experimental da Universidade do Tocantins, no Projeto Rio Formoso, na entressafra do arroz de 1995. As cultivares utilizadas foram EMGOPA 308, DOKO RC e EMBRAPA 31 (BR 81 ), todas de ciclo médio, semeadas em 28/06/1995, seguindo as recomendações oficiais para a cultura. O delineamento estatístico foi o de bloco ao acaso com três tratamentos (3 cultivares) e quatro repetições (4 blocos), sendo as parcelas formadas por dez fileiras de dez metros de comprimento, separadas por carreadores de dois metros. A partir de 25 dias, após o plantio, foram realizados levantamentos semanais, utilizando o pano de batida (3 batidas/ parcela) até o final do ciclo da cultura. $\mathrm{O}$ número de indivíduos coletados no período vegetativo e reprodutivo das cultivares (Fehr \& Calviness, 1979) foram 
transformados $(\sqrt{x+0,5})$ e submetidos a análise de variância com as médias comparadas pelo teste Tukey ao nível de $5 \%$ de probabilidade.

\section{RESULTADOS E DISCUSSÃO}

As principais lagartas desfolhadoras que ocorreram na cultura da soja, em ordem decrescente de abundância foram: Anticarsia gemmatalis, Hedylepta indicata (Fabricius, 1754) e Chrysodeixis includens (Walker, 1754) Foi verificada a predominância de $A$. gemmatalis sobre as demais, o que tem sido constatado em praticamente todos os levantamentos realizados em soja, em vários Estados do Brasil (Venzon \& Martins Filho, 1995). Apesar da ocorrência em praticamente todo o ciclo da cultura, esta espécie esteve presente em baixissima incidência (Fig. 1) e não diferindo significativamente entre as cultivares, tanto no período vegetativo como no período reprodutivo (Tab. 1). A flutuação populacional de $A$. gemmatalis apresentou picos populacionais relativamente baixos nas cultivares (Fig. 1),sendo que os baixos índices desta praga coincidiram com os maiores picos populacionais de Cycloneda sanguinea (Linnaeus, 1763) e Geocoris sp. (Fig. 4 e 5 ), que podem ter sido responsáveis pelo controle biológico.

Entre as espécies de pentatomídeos de importância econômica, foi constatada a ocorrência de Piezodorus guildinii, apresentando diferença significativa entre as cultivares durante o periodo vegetativo (Tab. 2). Na cultivar EMBRAPA 31 (BR 81), notou-se menor índice de ocorrência, seguida da cultivar Doko RC (Fig. 2). Durante o período reprodutivo não foi verificado diferença significativa entre as cultivares (Tab. 2). Os maiores picos populacionais não atingiram o nivel de controle ( $\mathrm{NC}=4$ percevejos/pano de batida) no caso de produção de grãos.

A ocorrência de $P$. guildinii, seguida de $E$. heros, tem sido citada em alguns trabalhos de levantamentos (Barcelos, \& Reis, 1978; Correia,

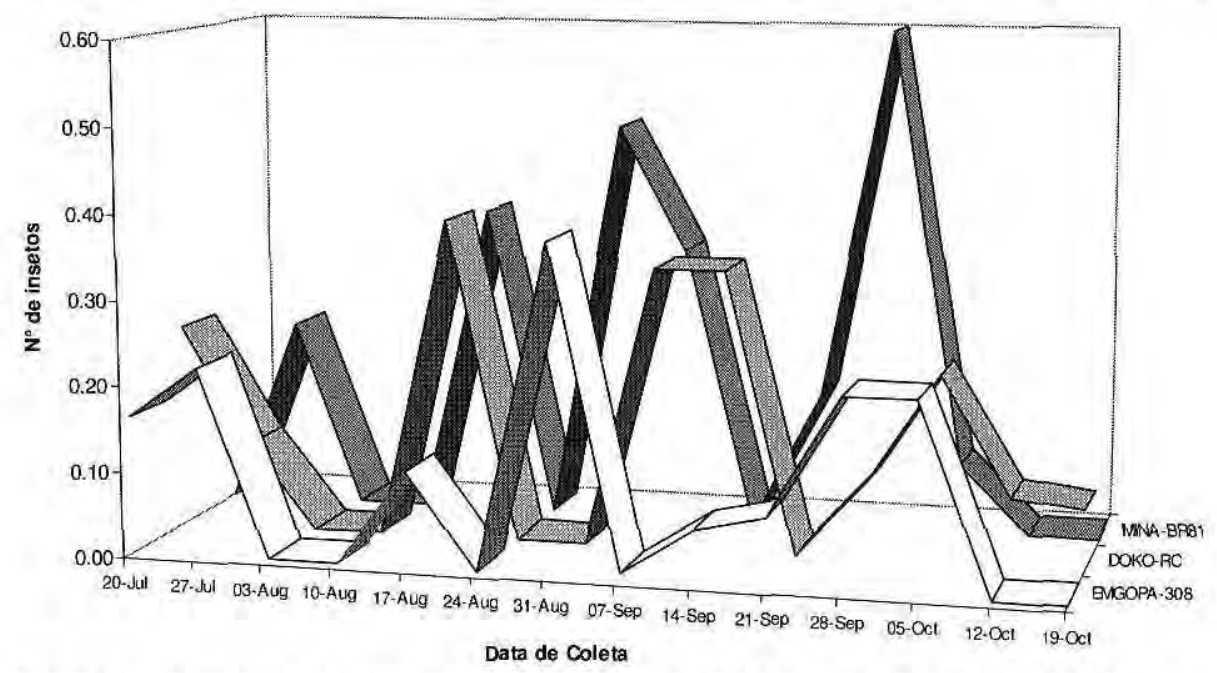

Figura 1. Ocorrencia de Anticarsia gemmatalis, coletada em $6 \mathrm{~m}$, pelo pano de batida, em 3 cultivares de soja. Formoso do Araguaia - TO. 1995. 
Tabela 1. Número médio' de Anticarsia gemmatalis, coletadas em $6 \mathrm{~m}$ pelo pano de batida, em 3 cultivares de soja. Formoso do Araguaia-TO, 1995.

\section{Estádio Fenológico}

Cultivares

Vegetativo Reprodutivo

EMGOPA 308

$0,08 \mathrm{a}$

$0,09 \mathrm{a}$

DOKO RC

$0,10 a$

$0,07 a$

EMBRAPA 31 (BR81)

$0,15 a$

$0,12 a$

$\mathrm{CV}(\%)$

16,23

16,25

' Dados originais, para análise foram devidamente transformados. Médias seguidas de mesma letra nas colunas năo diferem entre si, pelo teste de Tukey, ao nivel de $5 \%$ de probabilidade.

Tabela 2. Número médio' de Piezodorus guildinii, coletados em $6 \mathrm{~m}$ pelo pano de batida, em 3 cultivares de soja. Formoso do Araguaia-TO, 1995.

Cultivares

Estádio Fenológico

Vegetativo Reprodutivo

\begin{tabular}{lll}
\hline EMGOPA 308 & $0,42 a$ & $0,52 a$ \\
DOKO RC & $0,35 a$ & $0,65 a$ \\
EMBRAPA 31 (BR81) & $0,12 b$ & $0,24 a$
\end{tabular}

CV $(\%)$

15,50

11,12

'Dados originais, para análise foram devidamente transformados. Médias seguidas de mesma letra nas colunas não diferem entre si, pelo teste de Tukey, ao nivel de $5 \%$ de probabilidade.

1982; Leite \& Lara, 1985) e relatadas como espécies mais importantes para o Estado de Goiás (Corrêa et al., 1977; Prado et al., 1982).

Borges (1992), citado por SosaGomez et al. (1993) relata a importância do complexo dos coleópteros desfolhadores, como causadores de grandes desfolhas em soja no Estado de Mato Grosso. Em outros Estados, alguns autores têm-se referido apenas à sua ocorrência (Lorenzato et al., 1979; Prado et al.., 1982; Salvadori \& Gomez, 1982). No Projeto Rio Formoso, os coleópteros desfolhadores ocorreram durante todo o ciclo de desenvolvimento das cultivares, com predominância de Cerotoma sp., cuja 


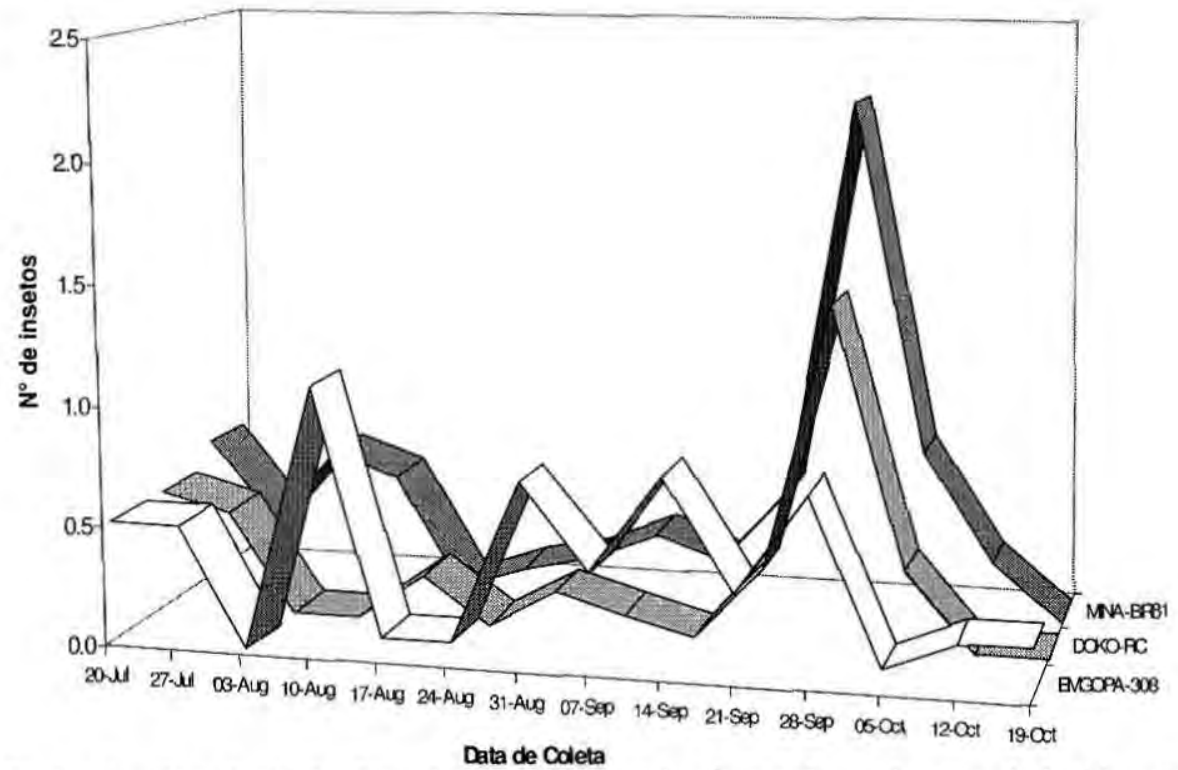

Figura 2. Ocorrência de Piezodorus guildinii, coletado em $6 \mathrm{~m}$, pelo pano de batida, em 3 cultivares de soja. Formoso do Araguaia - TO. 1995.

ocorrência apresentou diferença ocorrência de dois picos populacionais significativa entre as cultivares. O menor próximos ao período reprodutivo (Fig. 3 ), número de individuos coletados foi na cul- fato este também relatado por Corrêa $e t$ tivar EMGOPA 308 e Doko RC, no periodo vegetativo e reprodutivo, respectivamente (Tab. 3). Ainda com relação a espécie fọi constatada a al. (1977), Leite \& Lara (1985) e Venzon \& Martins Filho (1995).

Quanto aos inimigos naturais Cycloneda sanguinea, Geocoris sp.,

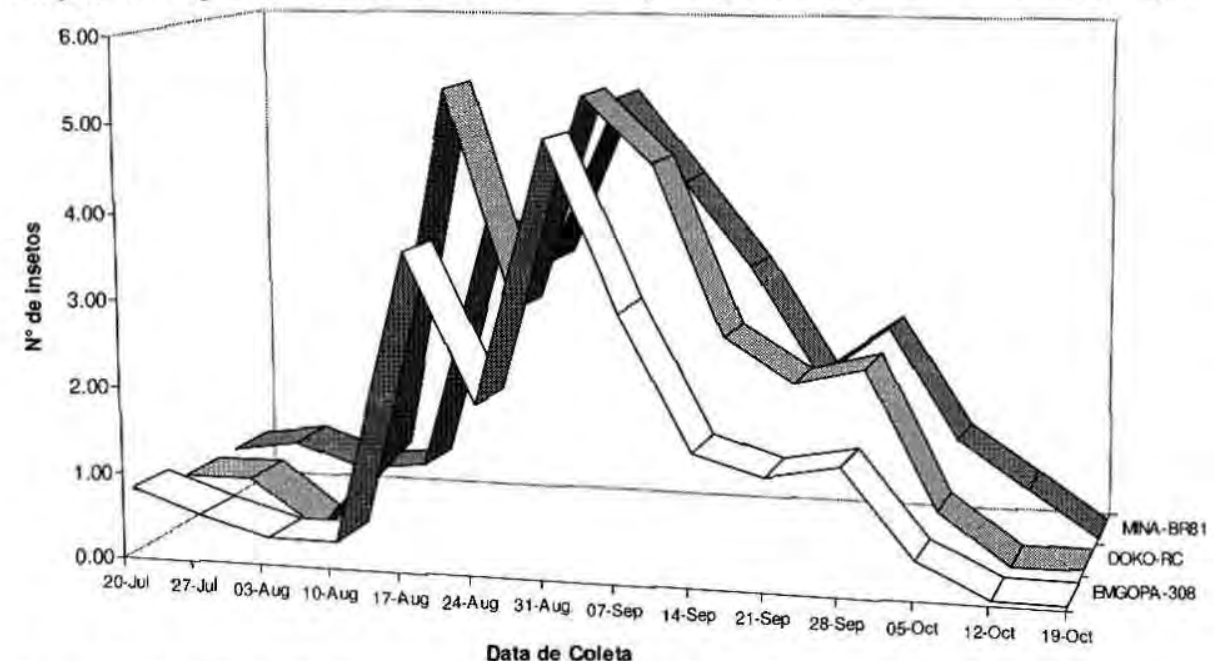

Figura 3. Ocorrência de Cerotoma sp., coletado em $6 \mathrm{~m}$, pelo pano de batida, em 3 cultivares de soja, Formoso do Araguaia - TO. 1995. 
Tabela 3. Número médio' de Cerotoma sp., coletadas em $6 \mathrm{~m}$ pelo pano de batida, em 3 cultivares de soja. Formoso do Araguaia-TO, 1995.

Estádio Fenológico

Cultivares

Vegetativo Reprodutivo

EMGOPA 308

$1,34 b$

$1,58 \mathrm{a}$

DOKO RC

$2,66 a$

$1,30 \mathrm{~b}$

EMBRAPA 31 (BR81)

$2,16 a$

$1,90 \mathrm{a}$

CV (\%)

7,28

6,04

- Dados originais, para análise foram devidamente transformados. Médias seguidas de mesma letra nas colunas năo diferem entre si, pelo teste de Tukey, ao nivel de $5 \%$ de probabilidade.

Lebia sp. foram as espécies mais abundantes. Os predadores $C$. sanguinea (Fig. 4) e Geocoris sp. (Fig. 5) apresentaram os maiores picos populacionais durante o estádio vegetativo da cultura, enquanto que Lebia sp. (Fig. 6) apresentou o maior número de indivíduos coletados no período reprodutivo.

\section{CONCLUSÕES}

1. A flutuação populacional de Anticarsia gemmatalis apresentou picos populacionais relativamente baixos, não apresentando diferença significativa entre as cultivares.

2. Ocorreram em menor abundância as lagartas desfolhadoras Hedylepta indicata e Chrysodeixis includens;

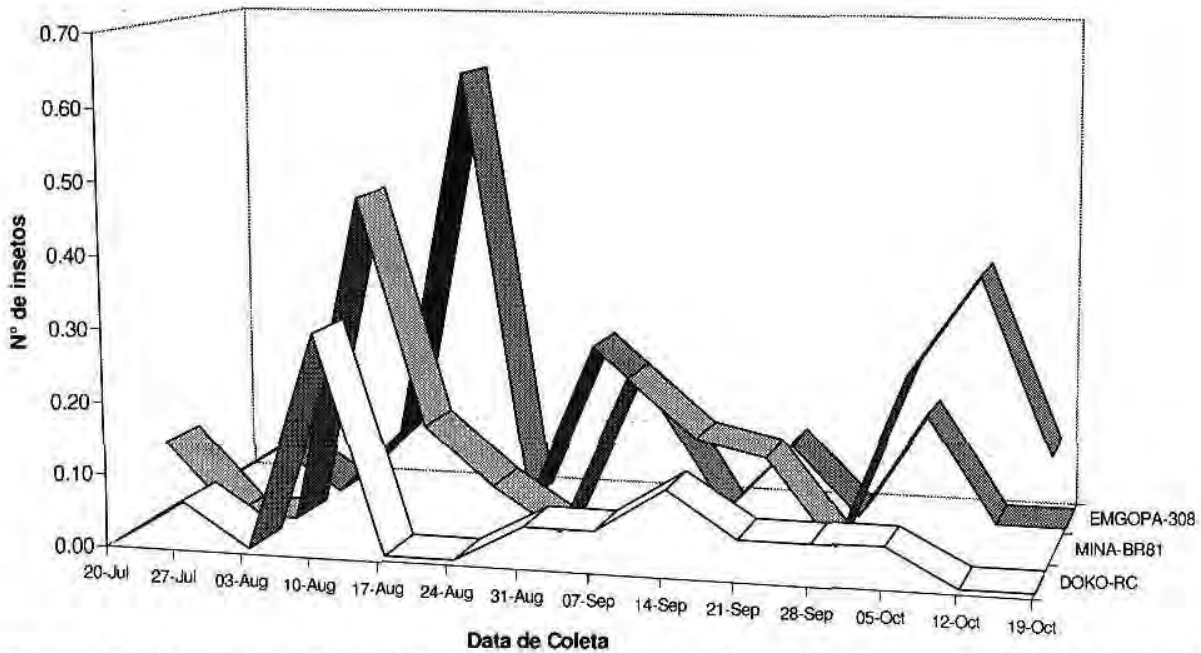

Figura 4. Ocorrência de Cycloneda sanguinea, coletada em $6 \mathrm{~m}$, pelo pano de batida, em 3 cultivares de soja. Formoso do Araguaia - TO. 1995. 


$$
\text { Alan }
$$




\section{AGRADECIMENTOS}

Ao CNPq/PIBIC pela concessão de bolsa de Iniciação Científica.

\section{Bibliografia Citada}

Barcelos, A.C.; Reis, P.R. 1978, Levantamento populacional de insetos na cultura da soja, na região do Triângulo Mineiro. Empresa de Pesquisa Agropecuária de Minas Gerais, Projeto Soja: Relatório76/77. EPAMIG/ ESAL/UFMG/UFV/EMBRAPA, Belo Horizonte, Minas Gerais. p. 13-15.

Borkert, C.M.; Yorinori, J.D.; Corrêa-Ferreira, B.S.; Almeida, A.M. R.; Ferreira, L.P; Sfredo G.J. 1994. Pragas: Diagnóstico e Controle. Informações Agronómicas 66. Potafos, Piracicaba, São Paulo. p. 13-14.

Corrêa, B.S.; Panizzi, A.R.; Newman, G.G.; Turnipseed, S.G. 1977. Distribuição geográfica e abundância estacional dos principais insetos-pragas da soja e seus predadores. Anais da Sociedade Entomológica do Brasil, 6(1): 40-50

Correia, A.C.B. 1982. Manejo de pragas da soja. Informe Agropecuário, 8(94): 47-56

Fehr, W.R. ; Caviness, C.E. 1979. Stages of Soybean development. Iowa State University, Ames, USA. 12p.

Gazzoni, D.; Oliveira, E. B.; Corso, I.C.; Ferreira, B.S.C.; Vilas Bôas, G.L.; Moscardi, F.; Panizzi, A.R. 1988. Manejo de Pragas da Soja. EMBRAPA/ CNPSo, Londrina, Paraná. 44p.
Leite, L.G.; Lara, F.M. 1985. Flutuação populacional de insetos e inimigos naturais associados à cultura da soja em Jaboticabal, SP, Anais da Sociedade Entomológica do Brasil, 14(1): 45-57

Lorenzato, D.; Gonçalves, H.M.; Sechin, J. 1979. Abundância estacional e efeito de inseticidas sobre espécies fitófagas da soja (Glycine max (L.) Merril) e seus inimigos naturais. Agronomia Sulriograndense, 15(1): 53-75

Prado, P.C.N.; Cunha, H.L; Silva, A.L. 1982. Ocorrência dos principais insetos-pragas da soja e seus inimigos naturais em Santa Helena de Goiás, GO. Anais do Seminário Nacional de Pesquisa de Soja, EMBRAPACNPSo, Londrina, Paraná. p. 111-139.

Salvadori, J.R. ; Gômez, S.A. 1982. Abundância estacional de insetos-pragas da soja e seus inimigos naturais em Dourados, MS. Anais do Seminário Nacional de Pesquisa de Soja, EMBRAPA-CNPSo, Londrina, Paraná. p.17-50.

Sosa-Gomez, D.R.; Gazzoni, D.L.; CorrêaFerreira, B.; Moscardi, F, 1993. Pragas de soja e seu controle. In: Arantes, N.E.; Souza, P.I.,de M. (Eds). Cultura da Soja nos Cerrados. Potafos, Piracicaba, São Paulo. p. 299-331.

Venzon, M.; Martins Filho S. 1995. Abundância de insetos-praga e seus inimigos naturais na cultura de soja no Triângulo Mineiro. Agricultura Tropical, 1(1): 79-91 